\title{
Supercapacitors using Pure Single-walled Carbon Nanotubes
}

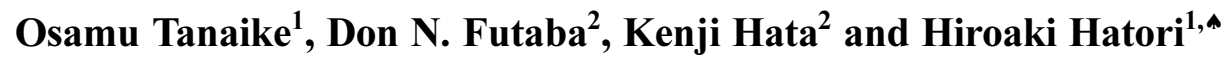 \\ ${ }^{1}$ Energy Technology Research Institute, Advanced Industrial Science and Technology (AIST), 16-1 Onogawa, Tsukuba 305-8569, Japan \\ ${ }^{2}$ Nanotube Research Center, AIST, 1-1-1 Higashi, Tsukuba 305-8565, Japan \\ ‘e-mail: h.hatori@aist.go.jp \\ (Received February 20, 2009; Accepted May 31, 2009)
}

\begin{abstract}
The excellent and characteristic capacitor performance of pure single-walled carbon nanotubes (SWNTs), which differ from conventional activated carbon electrodes, is reported. SWNTs with little bundling showed higher specific capacitance than activated carbons. High operating voltage can be expected for pure SWNTs without metal contamination and graphene edge structure.
\end{abstract}

Keywords : Carbon nanotubes, Capacitors, Electrochemical behaviors

\section{Introduction}

High performance electric double layer capacitors (EDLCs) which are generally known as "supercapacitors" have aroused much interest as electrical energy storage devices because high power density devices have huge market in the fields of hybrid vehicles, mobile instruments and so on. Supercapacitors have several excellent advantages when compared to secondary batteries; quick charge/discharge, long cycle life, high stability for operation, good acceptability to environment, etc. In order to realize supercapacitors with excellent and characteristic performance for commercialization, selection of electrode materials becomes an ultimately important subject to be resolved.

Single-walled carbon nanotubes (SWNTs) have high surface area theoretically up to $2600 \mathrm{~m}^{2} / \mathrm{g}$. The material is considered to be ideal for capacitor electrodes and hence many authors have reported the EDLC properties[1-3]. However, the intrinsic performance of SWNTs has not been clarified because the SWNTs used in the previous attempts contain considerable amounts of impurities and defects and have low surface area because of their bundle structure. Hata et al.[4] have reported a highly efficient synthesis method of impurity-free SWNTs. The "Super growth" method named gives a dense and vertically aligned SWNT forests (SGSWNTs) with millimeter-scale height. The SG-SWNTs can be easily separated from the catalyst, providing nanotube material with carbon purity above $99.98 \%$. Preliminary experiments on densely packed SG-SWCNT capacitor electrodes have proven compact, high power delivery, and high-energy-density storage, and have revealed the potential of SG-SWCNTs for the active material of next generation supercapacitors[5]. Here, we report an excellent and charac- teristic capacitor performance, attributable to the intrinsic nature of the SWNTs, which is distinct from activated carbon electrodes used in commercialized EDLCs.

\section{Experimental}

The preparation method of SG-SWNTs used is in the references[4]. A stand-alone SWNT sheet with BrunauerEmmett-Teller (BET) surface area more than $1100 \mathrm{~m}^{2} / \mathrm{g}$ can be easily pealed from the substrate used for CVD growth. The nanotubes are basically closed ones and the surface area of SG-SWNTs is of the outer surface. It is noteworthy that more than $80 \%$ of the theoretical value of the outer surface area $\left(1300 \mathrm{~m}^{2} / \mathrm{g}\right)$ is "isolated" from the surface of the other tubes, that is to say, SG-SWNTs have wide bundle-free surface. Commercially available activated carbons for EDLC (YP17, Kuraray Co., Ltd., BET surface area $1640 \mathrm{~m}^{2} / \mathrm{g}$ ) were used for comparison. The electrode sheets of activated carbon, YP17, with the thickness of $0.1 \mathrm{~mm}$ were prepared by mixing and kneading with $10 \mathrm{wt} \%$ poly(tetrafluoroethylene) binders and $10 \mathrm{wt} \%$ carbon black and then by hot-pressing.

The electrochemical measurements, cyclic voltammogram and galvanostatic charge/discharge, were performed by VMP2 potentiostat/galvanostat (Bio-Logic). Specific capacitance was determined from galvanostatic charge/discharge measurements according to standard RC-2377 of Electronic Industries Association of Japan (EIAJ). Discharge curves were measured by using two electrode cell system at a current density against the total weight of active materials in two electrodes, $\mathrm{I}=50 \mathrm{~mA} / \mathrm{g}$, after charging at the prescribed potential for $30 \mathrm{~min}$. The discharge capacitance (C) was calculated by the following equation, $\mathrm{C}=\mathrm{I} \times \Delta \mathrm{t} / \Delta \mathrm{V}$, where $\Delta \mathrm{t}$ and $\Delta \mathrm{V}$ are the time 


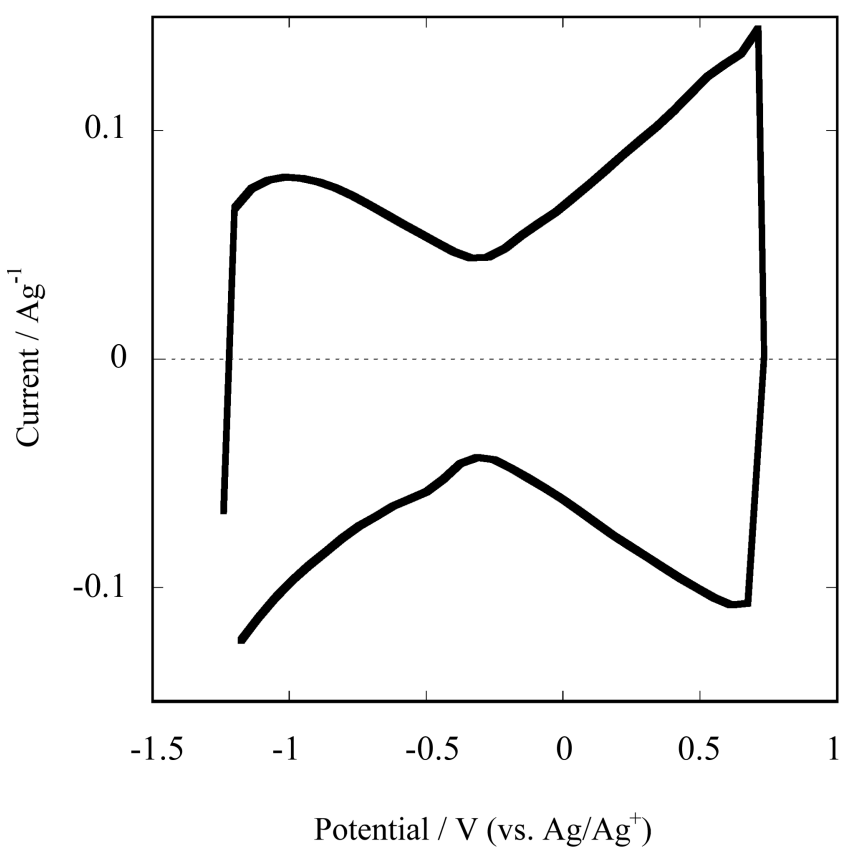

Fig. 1. Cyclic voltammograms in three electrode cell systems (scan rate: $1 \mathrm{mV} / \mathrm{s}$ ).

variation and the voltage variation between the $40 \%$ and $80 \%$ of the initial voltage, respectively.

\section{Results and Discussion}

The electrochemical properties of SG-SWNTs as prepared were evaluated in $1 \mathrm{M}$ tetraethylammonium tetrafluoroborate $\left(\mathrm{TEABF}_{4}\right)$ in propylene carbonate, the most popular electrolyte used for commercialized EDLCs. Cyclic voltammogram $(\mathrm{CV})$ measured by three electrode cell system gives a profile of butterfly shape as shown in Fig. 1. The current increased symmetrically to both the negative and positive sides from the center $\left(-0.3 \mathrm{~V}\right.$ against $\mathrm{Ag} / \mathrm{Ag}^{+}$reference) where the absolute value of current is minimum. Their characteristic mechanism of energy storage which differs from activated carbon electrodes has been reported[6]. The electronic conductivity of the SG-SWNT sheet is lowest around $-0.3 \mathrm{~V}$ in the electrolyte, where the current is lowest in $\mathrm{CV}$ profile in Fig. 1. The conductivity increases symmetrically both at the higher and lower potentials than $-0.3 \mathrm{~V}$, and the maximum conductivity become more than ten times as high as the minimum value at $-0.3 \mathrm{~V}$. SWNTs has characteristic electronic structure, which is symmetric and amphoteric as electron donor and acceptor, depending on the chirality. The energy storage mechanism is electrochemical doping similar to conductive polymers. Theoretical calculation study[7] has predicted the relation between SWNT's capacitive behavior and the electronic structure.

Galvanostatic discharge profiles measured by two electrode

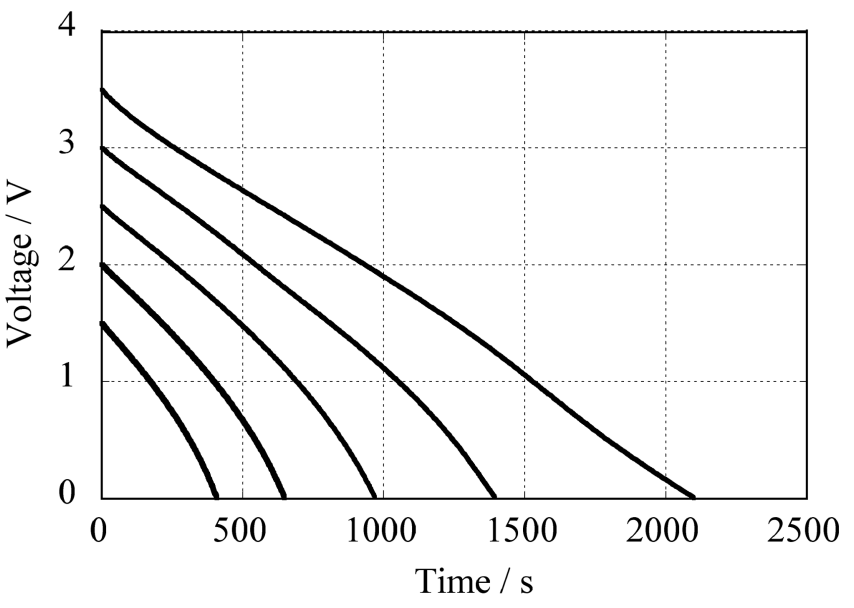

(a)

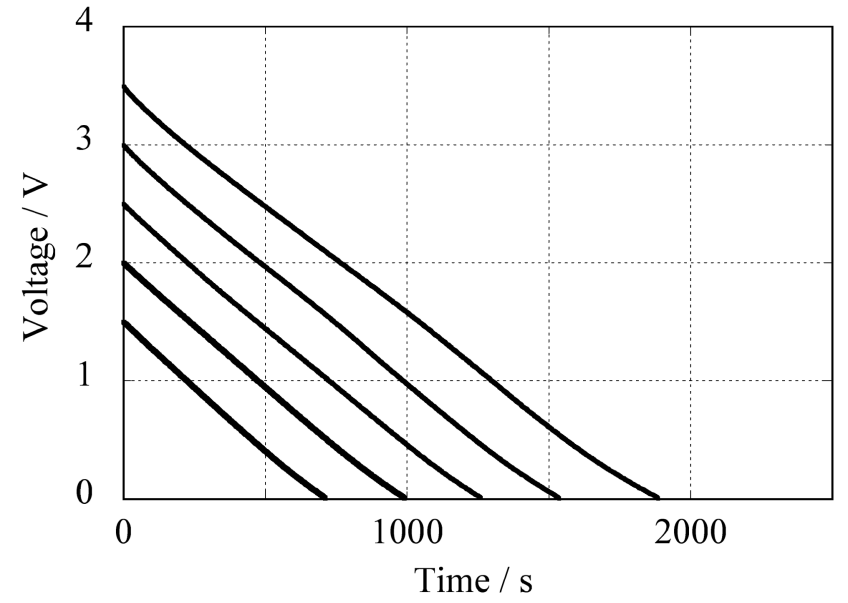

(b)

Fig. 2. Discharge profiles of (a) SG-SWNT and (b) YP17 measured by two electrode cells. The profiles were measured after charging for $30 \mathrm{~min}$ at prescribed cell voltages.

cells are shown in Fig. 2. The discharge measurements were performed after charging at the prescribed voltages for $30 \mathrm{~min}$. Because of the potential dependence by electrochemical doping, the discharge profiles of SG-SWNTs are parabolic and the slope becomes steeper at low range of cell voltage in comparison with the linear ones of YP17. The profile shows that the specific capacitance of SG-SWNTs decreases at low voltage. However, stored energy at the low voltage range is not important for capacitor cells because the amount of energy is proportionate to the square of cell voltage, and hence the cells are usually operated at relatively high voltage in practical use.

The specific capacitance determined from the discharge profiles is plotted against charging voltages in Fig. 3. The capacitance of activated carbon electrodes is basically independent of the voltages, but the higher the charging voltage is, the larger the SG-SWNT capacitance. Although the BET surface area of YP17 $\left(1640 \mathrm{~m}^{2} / \mathrm{g}\right)$ is higher than that of SG- 


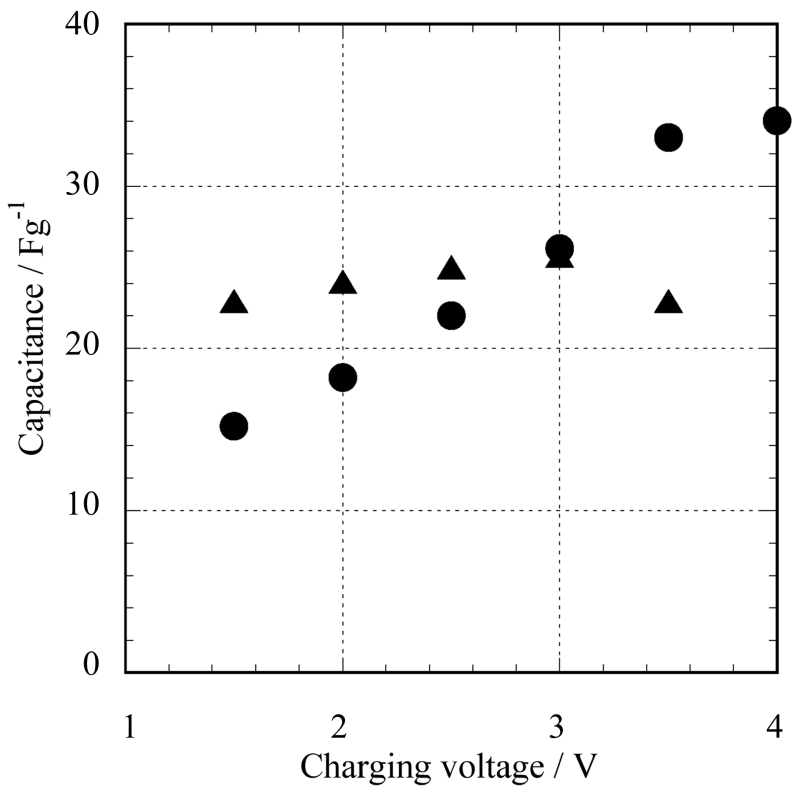

(a)

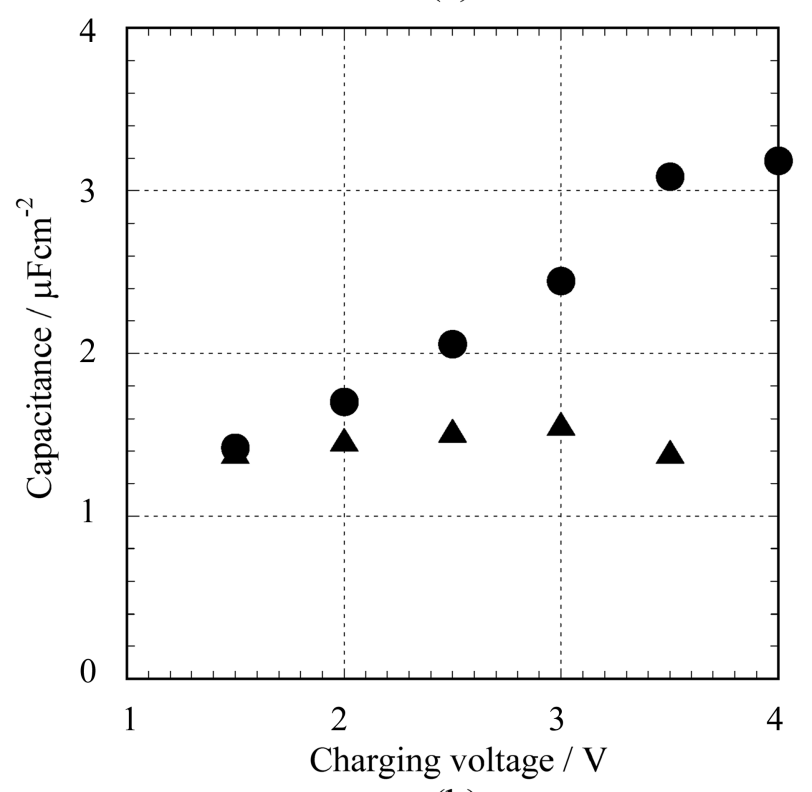

(b)

Fig. 3. Charging-voltage dependence of specific capacitance (a) per weight and (b) surface area of electrode materials; SGSWNT, $\Delta$ YP17.

SWNTs $\left(1100 \mathrm{~m}^{2} / \mathrm{g}\right)$, their values of capacitance per unit weight are the same at the charging voltage of $3 \mathrm{~V}$ (Fig. 3a). As a result, the capacitance per unit surface area of SGSWNTs becomes 1.5 times higher than that of YP17 (Fig. $3 \mathrm{~b})$. It is known that conductive polymers such as polyanilines and polythiophenes have high energy density as electrode materials, but the power density is limited because of slow ion diffusion in packed polymer chains. In case of bundled SWNTs, the nanotube surface inside of bundle is inactive until the ions can introduced between tubes by some intercalation reactions which require severe polarization conditions. Actually, bundled SWNTs such as HiPco nanotubes showed low capacitance because of their limited surface area even after the treatment of tube opening[3]. On the other hand, SG-SWNTs have high outer surface area exposed to the electrolyte and show high capacitance in practice as demonstrated in Fig. 3. In addition, the diffusion of ions occurs readily even in densely-packed SG-SWNTs having porous structure among tubes[5].

As shown in Fig. 3, the capacitance of SG-SWNT increased at the voltage more than $3.5 \mathrm{~V}$, though the performance of YP17 electrodes becomes worth or lost at the range. It is noteworthy that SG-SWNTs, the metal-free SWNTs, achieved high operating voltage than that of commercialized activated carbons for capacitor electrode which requires removal of metal contaminations causing catalytic electrolyte decomposition. We believe that a wide potential window on SWCNT surface with less graphene edge structure, being inactive for the decomposition of electrolyte, also contributes to the high operating voltage. Increase of charging voltage improve both energy and power densities of capacitor cells, being proportionate to the square of the charging voltage.

\section{Conclusions}

Pure SWNTs exposing wide bundle-free surface to the electrolyte are promising materials as supercapacitor electrodes because they possess both the strengths of porous carbons and conductive polymers; high power density because of the excellent conductivity of nanotubes and easy transportation of ions in the porous structure among nanotubes, and high energy density due to the doping mechanism. In addition, high operating voltage, attributable to the purity of SG-SWNT electrodes and a wide potential window of graphene with less edge structure, is absolutely advantageous from the application viewpoint.

\section{Acknowledgment}

We are grateful for partial support by the New Energy and Industrial Technology Development Organization (NEDO).

\section{References}

[1] Liu, C.; Bard A. J.; Wudl F.; Weitz I.; Heath J. R. Electrochem., Solid-State Lett. 1999, 2, 577.

[2] Frackowiak, E.; Jurewicz K.; Delpeux S.; Beguin F. J. Power Sources 2001, 97, 822.

[3] Shiraishi, S.; Kurihara, H.; Okabe, K.; Hulicova D.; Oya, 
A. Electrochem. Comm. 2002, 4, 593.

[4] Hata, K.; Futaba, D. N.; Mizuno, K.; Namai, T.; Yumura, M.; Iijima, S., Science 2004, 306, 1362.

[5] Futaba, D. N.; Hata, K.; Yamada, T.; Hiraoka, T.; Hayamizu, Y.; Kakudate, Y.; Tanaike, O.; Hatori, H.; Yumura, M.; Iijima, S. Nature Materials 2006, 5, 987.
[6] Kimizuka, O.; Tanaike, O.; Yamashita, J.; Hiraoka, T.; Futaba, D. N.; Hata, K.; Machida, K.; Suematsu, S.; Tamamitsu, K.; Saeki, S.; Yamada, Y.; Hatori, H. Carbon 2008, 46, 1999.

[7] Heller, I.; Kong, J.; Williams, K. A.; Dekker, C.; Lemay, S. G. J. Am. Chem. Soc. 2006, 128, 7353. 